\title{
Modeling AND ANAlysis of Cloud COLLABORATIVE COMMERCE
}

\author{
Hussein Al-Bahadili ${ }^{1}$, Awad Al-Sabbah ${ }^{2}$, and Muhammad Abu Arqoub ${ }^{1}$ \\ ${ }^{1}$ Faculty of Information Technology, Petra University, Amman, Jordan \\ hbahadili@uop.edu. jo, abu-arqoub@uop.edu. jo \\ ${ }^{2}$ Faculty of Information Technology, Middle-East University, Amman, Jordan \\ awadsabbaheyahoo.com
}

\begin{abstract}
Cloud computing has the potential to be particularly suitable for collaboration commerce (c-commerce) because it generally requires less extensive customization, development, integration, operation, and maintenance than other computing resources. However, upgrading c-commerce IT infrastructure, to run on cloud computing, faces a number of challenges, such as lack of effective design and implementation models. This paper describes and evaluates the performance of a new model of c-commerce that utilizes the evolving cloud computing technologies; as a result of that it is referred to as cloud collaborative commerce (cc-commerce) model. The model consists of six main components, these are: client, provider, auditor, broker, security and privacy, and communications network. The new cc-commerce model is used to develop a simple and flexible Web-based test tool, namely, the cc-commerce test (3CT) tool. The performance of the new model is evaluated by measuring the response times for four different configurations using the 3CT tool. The results obtained demonstrate that the cc-commerce model performs faster than equivalent $c$ commerce models.
\end{abstract}

\section{KEYWORDS}

Cloud Computing; collaborative commerce; cloud commerce; electronic commerce; cloud collaborative commerce.

\section{INTRODUCTION}

Electronic commerce (e-commerce) is one of the fastest growing applications for the Internet, which represents the sales aspect of electronic business (e-business) [1]. E-commerce is about performing transactions on the Web, such as purchasing and selling products, procurement, supply chain management, and training. While these fields have evolved somewhat independently over the past few decades, they have a lot to contribute to each other, i.e., collaborate. Organizations have realized that effective collaboration is a key to knowledge management and good knowledge management practices are essential for a successful organization; therefore, in recent years, many organizations are willing to go beyond private e-commerce and work collaboratively with other organizations. In other words, carry-out transactions, as well as share and collaborate on activities and projects using the Web to go beyond organizational boundaries, while remain autonomous [2]. This new form of collaboration is known as collaborative commerce (c-commerce) [3].]

C-commerce enables organizations to share information, and at the same time should provide all means to protect their privacy as well as all sensitive information. Furthermore, organizations 
usually build their resource management systems (RMSs) independently; therefore, it is highly expected to have disparate heterogeneous RMSs. Organizations usually run their RMSs on local area networks (LANs), which are accessed and interconnected through the Internet using wide area network (WAN) protocols and infrastructures [4]. Consequently, it is necessary to work on integrating these local systems to form federations that able to collaborate effectively.

Thus, many challenges must be addressed to meet users, organizations, and applications satisfactions and maintain satisfactory quality-of-service (QoS); such as: high access bandwidth, low-cost bit-rate, high network and link reliability, high efficiency, availability, scalability, security, etc. Adding to the above challenges are the high cost of the installation and operation of local RMSs. Also, all local resources need to be continuously maintained and updated [5].

Cloud computing is defined as the delivery of computing resources (hardware and software) as a service rather than a product, whereby shared resources, software, and information are provided to computers and other devices as a metered service over a network, typically the Internet [6]. Cloud computing providers deliver applications through the Internet, which are accessed from Web browsers running on desktop and mobile applications, while the business applications and data are stored on remote servers $[7,8]$. It is very interesting to perceive how c-commerce can benefit from this tremendous cloud computing technological development and relax the many challenges that are facing wider c-commerce promotion, and meet users', organizations', and applications' satisfactions and also maintain satisfactory QoS [9].

Nowadays, c-commerce information technology (IT) technologies have a tremendous effect on business success; therefore, many businesses give a great attention to this vital success factor. Many businesses, especially, small and medium businesses (SMBs) are often unable to possess their own suitable c-commerce IT technologies to enable them providing satisfactory services to meet their business and customers' needs on one hand, and to compete against large businesses on the other hand. This is as a result of: shortage of financial resources to build a suitable infrastructure, costs of software license for a start-up, shortage of IT experienced human resources, shortage of technical knowledge to maximize benefits of IT systems, and high operation and upgrading costs [10].

Moreover, c-commerce faces a number of challenges that must be carefully considered, such as: (1) IT technologies of all partners or participants must have equivalent performances, (2) internetworking infrastructures of the partners acquire a number of requirements, such as: highbandwidth, reliability, availability, etc., (3) IT and internetworking infrastructures must have a powerful information and network security, and (4) heterogeneous infrastructures of the partners may acquire a number of middleware applications $[9,10]$. Cloud computing platforms have the capacity to be particularly suitable for applications required collaboration between participating parties, such as those imposed by c-commerce applications. It generally requires less customization, development, integration, and installation, operation, and maintenance costs, than other enterprise applications [11].

This paper, first, develops a new c-commerce model that utilizes cloud computing concept as a platform for running c-commerce application, which is referred to as cloud-based collaborative commerce (cc-commerce). The cc-commerce components and the deployment configurations for the proposed model are described in details. Second, the performance of the new model is evaluated qualitatively and quantitatively. The performance is evaluated quantitatively in terms of the average response time and speedup factor.

This paper is developed into seven sections. Section 1 introduces the main theme and concept of the paper. The remaining part of the paper is organized as follows: Section 2 presents a background on c-commerce, traditional computing systems and cloud computing. Section 3 
provides a review on some of the furthermost recent and related work. A detail description of the new cc-commerce model is given in Section 4. The model implementation and performance measures are given in Section 5. Section 6 presents a description of four scenarios representing ccommerce and cc-commerce configurations that are performed to evaluate and compare the performance. Finally, in the Section 7, conclusions are drawn and a number of recommendations for future research and investigation are pointed-out.

\section{BACKGROUND}

This section provides some background on the main topics that are represent the core of this research, these are: c-commerce, traditional computing systems and cloud computing systems.

\subsection{Collaborative Commerce (C-Commerce)}

C-commerce is a form of e-business; conducting business on the Internet, not only buying and selling (e-commerce) but also servicing customers and collaborating with business partners. Ccommerce is conducted via inter-enterprise Internet connections and enables multiple enterprises to work interactively online to find ways to serve their customers and to solve business problems. $\mathrm{C}$-commerce are also defined as enterprise capable Web-based solutions that use the Internet to allow employees, customers, and suppliers to collaboratively develop, build, manage, sell, and support products throughout their life cycle $[5,7,8]$.

\subsubsection{C-Commerce Models}

In order for organizations to carry out c-commerce collaboratively, one needs suitable business models [12]. Seller to buyer relationships in c-commerce can take one of many forms; these are: tightly-coupled and loosely-coupled c-commerce. In tightly-coupled model, one party dominates the business practices and the others conform to them. Because the dominating party is so large, it is either the dominant seller or the dominant buyer in the market. Therefore, it has the power to dictate what system will be used to conduct the transaction. Tightly-coupled relationships can take the form of a one-to-one, a many-to-one, or one-to-many relationship.

Conversely, in loosely-coupled model, no party has the power to dictate the system used to conduct transactions. In loosely-coupled environment a company does not have one main supplier or customer, instead it may deal with whichever company it pleases, taking into account such things as price, value, and reputation. In order to complete the transaction, the companies must either constantly be changing their business practices to adapt to new partners or there must be some sort of mitigation system which can resolve differences between partners. This would allow a business to form a relationship with another with minimal effort or changes to their e-commerce infrastructure. However, the forming of such a mitigation system is not trivial and may require serious investment.

\subsubsection{The Vision of C-Commerce}

The main objectives for using c-commerce initiatives are to optimize return on IT investment, increase business agility, enhance the quality of service to the customer, and improve supply chain integration. Integrating c-commerce applications using current advanced standards and open technologies enable business partners to manage complex transactions, share their updated information, collaborate on product planning, communicate design ideas, and integrate their work frameworks [5]. C-commerce can help companies gain competitive advantages by connecting and automating processes with their partners; customers, and suppliers; reducing processing latencies; 
developing new capabilities that improve service levels while reducing costs; and making planning, design, and operational decisions dynamically based on real-time information [7].

In c-commerce, business processes interactions must be carefully managed among internal and external applications involved in the process to achieve optimized of collaborative business processes. There are different levels of integration in c-commerce, such as: exchange data via traditional means such as fax, phone, and mail; use EDI or email for point-to-point data interchange; utilize private or public exchanges to share business process information; and deploy Web services and process management tools to coordinate loosely coupled services into integrated inter-enterprise processes with real-time data sharing [13].

C-commerce technologies must support inter-operability because trading partners often use diverse heterogeneous systems. Consequently, solutions for c-commerce must be based on standard models to support loose coupling, autonomy, and flexibility on the one hand, as well as ensure trust, privacy, and security on the other hand. Unfortunately, till this moment, there are only few standard models and technologies that are considered specifically suitable for ccommerce, including simple object access protocol (SOAP) as a messaging standards for accessing software components in distributed applications; common document specification languages such as XML and ebXML (electronic business XML) for information exchange; and business process specifications such as business process modeling language (BPML) for frameworks description [5].

\subsubsection{Building Blocks of C-Commerce}

The main building blocks of a c-commerce are: knowledge management, e-commerce, and collaboration as shown in Figure 1. E-commerce assumes that following: sellers sell their products through the Web and at the same time buyers purchase these products; organizations have to work collaboratively to perform transactions, and consequently Web collaboration is an essential part of c-commerce. For example, groups of designers and manufactures have to collaborate with one another in order to come up with a new design or product. Knowledge management captures the best practices and capabilities from preceding transactions and makes use of them efficiently to carry out c-commerce $[12,14]$.

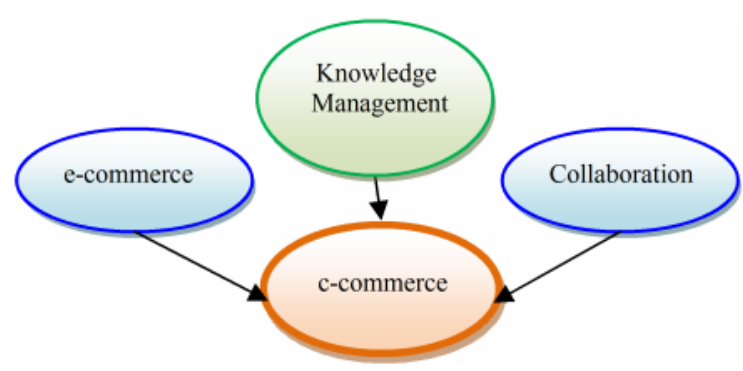

Figure 1. Building blocks of c-commerce.

\subsection{Traditional Computing Services}

Before we explain cloud computing, let us first review how IT solutions are typically delivered to businesses before the emergent of cloud computing. Two broad approaches can be identified to providing computing services; these are [15]: on-premises IT services and data canter IT services. On-premises IT is where the business purchases, installs and maintains all servers, software licenses, backup devices and telephony equipment which are housed on-site in the business 
office. Of course, technology is complicated so IT experts are always required to maintain the system operation at business or company site. Companies either hire on-staff IT employees or hire local IT services firms to perform this maintenance. Maintaining of the IT infrastructure is not an easy task, and very often the IT support staff is overloaded and subsequently the business has to wait long for IT support.

On-premises IT services demonstrate a number of advantages, these include: high local control, ability to customize services locally, local data storage, and can operate without Internet. On the other hand, they have many disadvantages, such as: requires specialized IT support staff, initial and operation capital cost intensive, difficult to upgrade and patch, unpredictable additional costs, difficult to maintain compliancy, and data center may not provide high availability [16].

Data center option is very often confused with cloud computing, where it is not cloud computing. This option allows companies to utilize computing resources available at a dedicated managed data center, which has many advantages over traditional on-premises systems, but, sometimes, it still requires the company to purchase servers, licenses and often pay the provider for maintaining their system or employ an internal IT staff. Managed data centers have many advantages over onpremises IT services, these include: provide state-of-the- art computing infrastructure; provide high-class data center facility and state-of-the-art network infrastructure; enable users to maximize the usage of their own networking assets to improve business's internal network operations; provide immediate increase in the network reliability and scalability; provides competent IT staffs for operating, monitoring and maintaining the system, give a continuous operation, continuous support, security, and flexibility; help the business developer to focus on their own core business, improve business core services for greater profitability by helping them moving away from daily managing IT infrastructure and problem solving; reduce IT cost and risks due to reduction in IT manpower requirement together with eliminating the need for retaining staff with high IT skills. On the other hand, they have many disadvantages, such as: difficult to upgrade and patch, unpredictable costs, high costs for managed services, and redundant cost if some systems that are still required on premises [16].

\subsection{Cloud Computing}

Cloud computing is a model of hardware and software deployment where the software application is hosted on extremely high performance hardware, as a service provided to customers across the Internet $[6,17]$. By eliminating the need to install and run the application on the customer's own servers or premises, the burden of software maintenance, ongoing operation and support has been removed. Resources (software and hardware) are paid for through a subscription instead of a license. Businesses no longer require to purchase and install high cost software or hardware assets on their premises. Computing becomes a predictable operating expense [10].

One of the main advantages of cloud computing is that users are able to leverage the economies of scale provided by the cloud service providers (CSPs) ability to purchase and manage repeatable processes and technologies. Providers typically focus on common services so they don't need to support (and pay for) a very diverse set of technologies and expensive labor force to maintain it. These costs are passed on to the subscriber. Unlike Web hosting solutions, there is only a limited number of CSPs. But fortunately all the major hardware and software brands of the world including Google and Microsoft are already providing cloud solutions. Major cloud service providers are HP, DELL, Amazon and IBM.

\subsubsection{Types of Cloud Computing Services}

Cloud computing users can expect to get three types of services from CSPs; these are [6]: 
(1) Cloud infrastructure as a service (IaaS): All required hardware to run a business is provided by CSPs and customers manage their application software.

(2) Could platform as a service (PaaS): A customer pays to the service provider to use their platform as their IT solution. For example, if you need E-mail system or database software for your business, you can use a third party's computing service that prove email and database solutions.

(3) Application software as a service (SaaS): If you only need to use a specific kind of software to get an output or to perform an analysis, then it is much cheaper to use that software service from a CSP rather than buying, installing and maintaining it.

\subsubsection{Benefits of Cloud Computing}

The main benefit of using cloud computing is saving cost in IT infrastructure installation and management; because in a cloud environment, users do not have to buy any hardware [19]. Users just need to buy the computing services from a CSP and those services can be accessed online. It is seen that to run a small and mid-size business, the majority of the investment are wasted in buying hardware and software. On the other hand, a cloud computing solution can save initial investment on hardware purchase, and thus help small business owners to spend more money on the core areas of business. Other benefits of cloud computing may include: lower capital expenditures, employees work anywhere and focus on business not technology, no upgrades concerns, instant implementations, lower upfront and operation and maintenance costs, pay only for the required services, guaranteed service-level agreements (SLAs), and predictable spending.

Start-up businesses usually see the cloud hosted services as a very attractive option. However, more experienced and large businesses look far beyond costs as a sole consideration, especially those operating in industries with strict legislation and compliance regulations, where they look at the following: third-parties are handling confidential data (i.e., information security), requirement of reliable Internet connection, redundancy, reliability and what will happen if the solution provider fails, a subscription service could be expensive over time and as business grows, the limited customization and integration with local systems could be an issue, and lack of full control over data and applications.

\subsection{Cloud Collaboration}

Cloud collaboration means sharing and co-authoring of information through the utilization of cloud computing, whereby information is uploaded to central cloud storage, where it can then be accessed by others. Cloud collaboration technologies have allowed users to upload, comment and collaborate on stored information and even amend it, evolving the information within the cloud. In the last few years, it has been realized that businesses have increasingly been switching to use cloud collaboration $[11,20]$, because it brings together new advances in cloud computing and collaboration that are necessary for businesses operating in the current globalized world.

Collaboration is defined as the ability of workers to work together concurrently on a particular task. In the past, most collaboration would have to be accomplished through face to face meetings. However, collaboration nowadays becomes more complicated as it may require collaboration of people from all over the world in real time on a variety of different types of information, using different technologies. While collaboration is still growing rapidly, it has been noted that the uptake of cloud collaboration services has reached a point where it is less to do with the ability of current technology, and more to do with the unwillingness of workers to collaborate. A recent report by Erica Regulates mapped out the reasons for workers unwillingness to collaborate more. These are: people don't like sharing their knowledge; people like using e- 
mail as their primary collaboration tool; people do not have motivation to change their behavior; teams do not have leaders who push for more collaboration; and senior managers are not actively involved in or do not support the team collaboration initiative.

Fortunately, many providers of cloud collaboration tools have involved heavily creating solutions to these problems, such as: integration of email alerts into collaboration software and the ability to see who is viewing the document at any time. All tools that are required by a team are put into a single piece of integrated software so users no longer have to rely on email based solutions.

While e-commerce enables organizations to carry out transactions, and knowledge management captures the knowledge of an organization, we still need a third component to enable organizations to work together and at the same time be autonomous, the third component is known as collaboration. Collaborative computing enables organizations to work together to complete a task or a collection of tasks. These tasks could vary from participating in solving a specific problem, or working on the design of a system. Several technologies including networking, distributed processing, and database systems, multimedia as well as several diverse disciplines such as organizational behavior and human computer interaction (HCI) have contributed significantly towards the development of collaborative computing. Early collaborative computing systems focused on small teams working together, while collaboration has taken on a larger dimension and scope with the advent of the Web and Internet technologies. Organizations can now collaborate across international boundaries, without making any a priori arrangements, and perform mutual and joint transactions in a near-real-time basis.

Cloud computing provides an alternative reliable and efficient approach to achieving business objectives that are enabled by collaboration solutions. Utilization of collaboration tools on the cloud are approved to be relatively has low-risk and high-return on investment. The fact is that some services available on the cloud already have a proven track record. They include Web conferencing services, such as Microsoft Live Meeting or WebEx. Most people have used these and other services and done so confidently for a long time on the cloud.

As cloud computing begins to take hold for companies considering new IT opportunities, the benefits of utilizing collaboration technologies as a service through the cloud become readily apparent. Cloud collaboration immediately opens options for access to new and cost-effective ways to address company goals and objectives. For example: being able to get communications and collaboration services up and running quickly, lower upfront deployment costs, ease of access, pay only for required and used software solutions, and access to updated software.

If used effectively, cloud collaboration enables organizations to capitalize on required technologies without the extensive, upfront capital expense that comes with the time-consuming installation and configuration of IT systems. With cloud, organizations need to determine which applications they must have and control, and which ones are prime targets for the cloud because they require less control. In essence, organizations must decide how much control over certain aspects of IT they actually need. Then they must compare how much financial value that control offers by being in-house compared to the economies of scale available on the cloud.

When considering cloud, organizations must know that partners, or vendors, they work with are credible. They should have proven processes to support an organization's needs. It's equally important to identify organizations with a track record of on-premise IT solutions. Those organizations should also have a clear cloud vision, strategy and offerings that demonstrate stability and long-term viability. If personal information is put into the cloud, legal requirements for transferring, storing and using information must be priory addressed. These requirements vary from jurisdiction to jurisdiction. 
International Journal on Cloud Computing: Services and Architecture (IJCCSA),Vol.3, No.1, February 2013

\section{Previous Work}

In this research, we mainly interested in three related topics: c-commerce, cloud computing, and cc-commerce. The research in c-commerce area includes business and IT aspects. Business aspect is beyond the scope of this paper. Therefore, in what follows we shall review some of the most recent and related work to c-commerce and applications of cloud computing in c-commerce. However, we have realized that very little efforts being focused on cloud computing performance evaluation for c-commerce applications may be mainly, because this is a very new area of research.

Over the last decade numerous efforts have been made in the area of c-commerce. Huang \& Fan [21] proposed an integrated solution for c-commerce including the collaborative strategy, model and platform. The reference processes and strategy of c-commerce is derived using a structural top-down analysis approach. According to the analysis and abstract of c-commerce behavior, the collaborative meta-model is put forward to support the description of complex collaborative relationship in c-commerce environment. On the foundation of collaborative meta model, the collaborative model of c-commerce can be established. Finally, the service oriented architecture (SOA) and model-driven architecture (MDA) based-platform for c-commerce is developed to enable value-added collaboration between partners by providing new technical solutions, best practices, and collaboration tools. The platform represents a virtual, model-driven and serviceoriented integration environment accessible to the involved companies within a heterogeneous ITinfrastructure.

Chen et al [5] proposed architecture for Web services enabled business process management (BPM) in c-commerce and provided technical insights into why Web services can enhance business process coordination. Also, they presented an implementation of a dynamic eprocurement application based on the proposed architecture. They concluded that with the advent of Web service standards and business process integration tools that support them, BPM systems enabled by Web services are empowering the development of more flexible and dynamic ccommerce.

Zhang et al [22] put forward a c-commerce model based on multi-agent system (MAS) technology that is characterized with the characteristics of autonomy, easy adaptation, flexibility and dynamic of agent technology. It can be used to describe the interaction relationship with partners and run on the cooperation among the different MAS layers. Many useful functions (e.g., automated learning, data analysis and mining, best solution selection, etc.) can be realized in this unified c-commerce system.

Buyya et al [18] defined the architecture for creating clouds with market-oriented resource allocation (MORA) by leveraging technologies such as virtual machines (VMs). They provided insights on market-based resource management strategies that encompass both customer-driven service management and computational risk management to sustain SLA-oriented resource allocation. In addition, they revealed their early thoughts on interconnecting clouds for dynamically creating global cloud exchanges and markets. Then, they presented some representative cloud platforms, especially those developed in industries, along with their current work towards realizing MORA of clouds as realized in Aneka enterprise cloud technology. Furthermore, they highlighted the difference between high performance computing (HPC) workload and Internet-based services workload. They also described a meta-negotiation infrastructure to establish global cloud exchanges and markets, and illustrated a case study of harnessing storage clouds for high performance content delivery. Finally, they concluded the need for convergence of competing IT paradigms to deliver the $21^{\text {st }}$ century vision. 
Chang \& Wang [23] explored the integration of internal and external business processes and the coordination of collaborative design teams. An initial qualitative investigation explores the practical applications of an Enterprise Information Portals (EIP) in an automobile company. A research model is then formulated and tested using a questionnaire survey of the R\&D department of a motor company in Taiwan. The results of the data analysis reveal that presence of EIPs can help a company realize the benefits of c-commerce. EIPs also can improve c-commerce performance by promoting the degree of integration of the enterprise process and by strengthening the process innovation and communication of collaborative design teams. Their results suggest that managers should reinforce important factors, including knowledge management tools, process integration, and the quality of design teams, in order to achieve success in c-commerce.

Li \& Xia [11] made a preliminary study on how to make cloud computing be applied in the ccommerce chain and presents the principles, ideas and analysis of the architecture to provide references on the construction of cloud computing-based c-commerce chain. C-commerce chain is a new business model with net-chain structure which is built based on mutual benefit and winwin strategy and it meets with the needs of cooperation in production and management between branches of the enterprise, between enterprises and customers and business to business such as: procurement, manufacturing, sales and after-sale services. It implements the effective planning and control on the business flow, logistics, capital flow and information flow involved in business operations and forms a strategic alliance body with core competitiveness. Li et al. showed that cloud computing-based c-commerce chain has five layers which are structural layer, basic resource layer, middleware layer, logic layer, and collaborative application layer. When the architecture of cloud computing-based c-commerce chain is being designed, it should be considered that the different characteristics of collaboration chains have different focuses. It is worth mentioning that the resource structure highlights the security system as the security management tools and related strategies are designed to keep cloud computing to deal with business tasks safely. However, they did not discuss any implementation or performance evaluation to their proposed model.

Chanchary \& Islam [9] explored the key requirements of e-commerce business model based on cloud computing and cited it as cloud commerce. Assuming these requirements, they made a comprehensive study on existing facilities for e-business patterns in Saudi Arabia to assess the readiness of this country to adopt with the emerging trend. They used a structured framework for this assessment that separated in three distinguishable forms such as architectural readiness, infrastructure readiness and process readiness. To summarize each stage of this framework, they followed a pattern of data collection that mainly included interviews and questionnaires.

Exposito et al [24] analyzed the main performance bottlenecks in HPC application scalability on the Amazon EC2 Cluster Compute platform: (1) evaluating the communication performance on shared memory and a virtualized 10 Gigabit Ethernet network; (2) assessing the scalability of representative HPC codes, the NAS Parallel Benchmarks, using an important number of cores, up to 512 ; (3) analyzing the new cluster instances (CC2), both in terms of single instance performance, scalability and cost-efficiency of its use; (4) suggesting techniques for reducing the impact of the virtualization overhead in the scalability of communication-intensive HPC codes, such as the direct access of the virtual machine to the network and reducing the number of processes per instance; and (5) proposing the combination of message-passing with multithreading as the most scalable and cost-effective option for running HPC applications on the Amazon EC2 Cluster Compute platform 


\section{THE NeW CC-COMMERCE MODEL}

This section presents an overview of the reference architecture of the new cc-commerce model, which identifies the major components, their activities and functions. The cc-commerce model is assumed to consist of six main components as depicted in Figure 2; these are: client, provider, auditor, broker, security and privacy, and communication network. Each component is an entity (a user or an organization) that participates in a transaction or process and/or performs tasks in cccommerce.

Table 1 lists and defines the components of cc-commerce reference architecture. Figure 3 illustrates the interactions among the components. A cc-commerce client may request cccommerce services from a cc-commerce provider directly or via a cc-commerce broker. A cccommerce auditor conducts independent audits and may contact the others to collect necessary information. The general activities of the components and the details of the architectural elements are discussed this section.

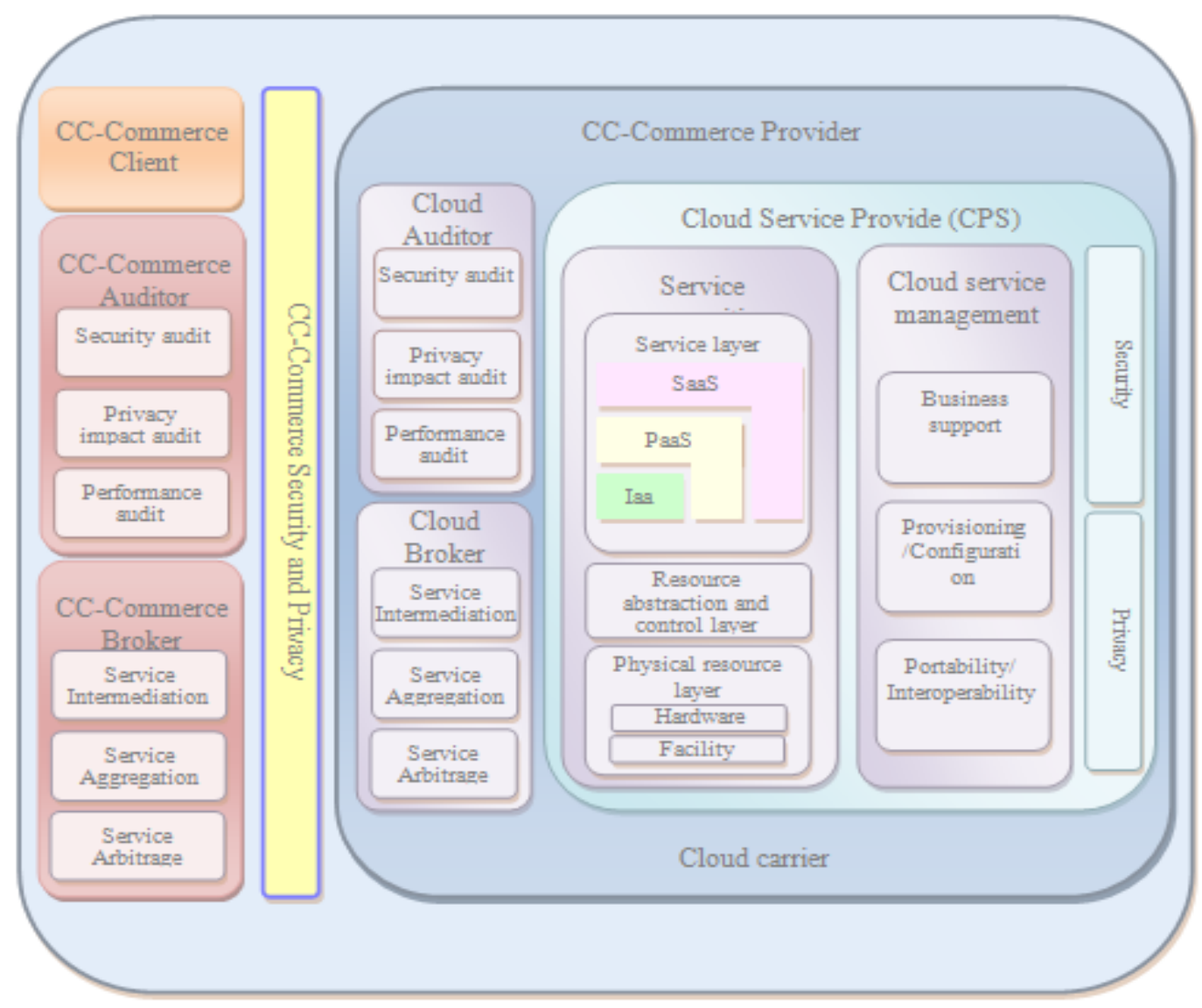

Figure 2. Architecture of the cc-commerce model. 
Table 1. Components of the cc-commerce model

\begin{tabular}{|l|l|}
\hline \multicolumn{1}{|c|}{ Component } & \multicolumn{1}{c|}{ Definition } \\
\hline Client & $\begin{array}{l}\text { A user/organization/entity that maintains a business relationship } \\
\text { with, and uses service provided by cc-commerce providers or } \\
\text { brokers. }\end{array}$ \\
\hline Provider & $\begin{array}{l}\text { A user/organization/entity responsible for making a service } \\
\text { available to interested parties. }\end{array}$ \\
\hline Auditor & $\begin{array}{l}\text { A party that can conduct independent assessment of cc-commerce } \\
\text { services, operations, performance, privacy, and security of the } \\
\text { cloud implementation. }\end{array}$ \\
\hline Broker & $\begin{array}{l}\text { A party/entity that manages the use, performance and delivery of } \\
\text { cc-commerce services, and negotiates relationships between } \\
\text { providers and clients. }\end{array}$ \\
\hline $\begin{array}{l}\text { Security and } \\
\text { Privacy }\end{array}$ & $\begin{array}{l}\text { A party/entity that is responsible for providing security and } \\
\text { privacy across the system components using standard and/or } \\
\text { specially developed protocols. }\end{array}$ \\
\hline $\begin{array}{l}\text { Communications } \\
\text { Network }\end{array}$ & $\begin{array}{l}\text { An intermediary that provides connectivity and transport of cc- } \\
\text { commerce services between cc-commerce clients to } \\
\text { providers/brokers. }\end{array}$ \\
\hline
\end{tabular}

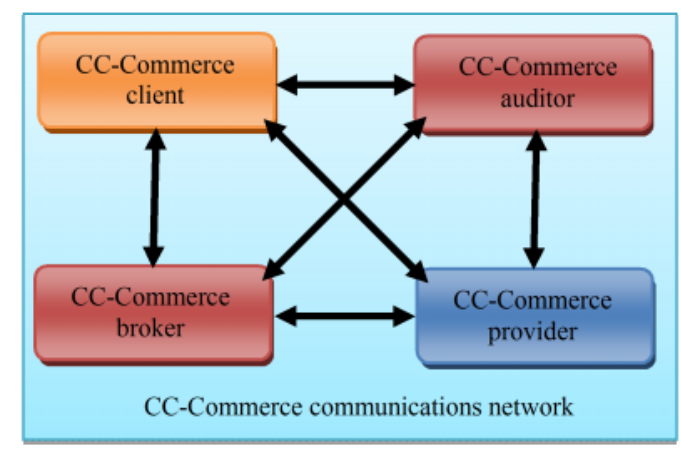

Figure 3. Interactions between the components in cc-commerce.

\subsection{Model Configurations}

The model can be configured and used in three different ways; these are:

(1) Configuration\#1: A cc-commerce may request service from a broker instead of contacting a provider directly. The broker may create a new service by combining multiple services or by enhancing an existing service. In this example, the actual providers are invisible to the client and the client interacts directly with the broker. This configuration is illustrated in Figure 4. 


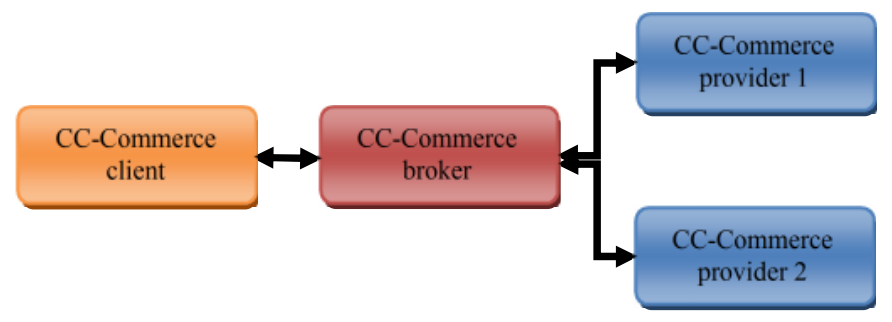

Figure 4. Interconnection in Configuration \#1.

(2) Configuration\#2: Communications network provides the connectivity and transport of cccommerce services from providers to clients. As illustrated in Figure 5, a provider participates in and arranges for two unique SLAs, one with a communications network (SLA2) and one with client (SLA1). A provider arranges SLAs with a communications network and may request dedicated and encrypted connections to ensure the cc-commerce services are consumed at a consistent level according to the contractual obligations with the clients. In this case, the provider may specify its requirements on capability, flexibility and functionality in SLA2 in order to provide essential requirements in SLA1.

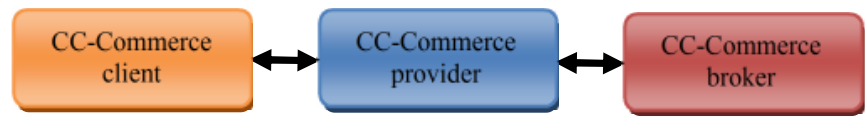

Figure 5. Interconnection in Configuration \#2.

(3) Configuration\#3: For a cc-commerce service, an auditor conducts independent assessments of the operation and security and privacy of the cc-commerce service implementation. The audit may involve interactions with both the client and the provider. Figure 6 shows the interconnection between the collaborating components.

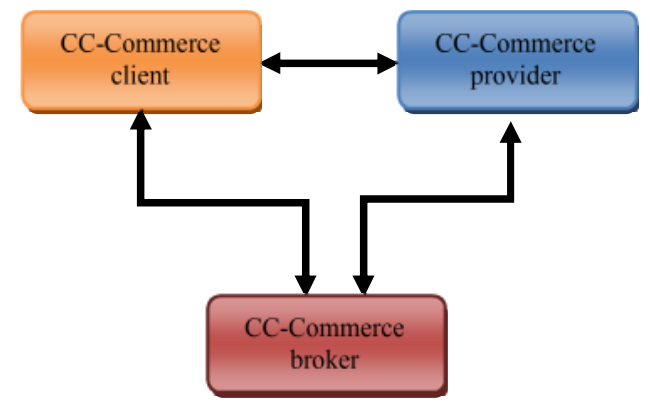

Figure 6. Interconnection in Configuration \#3.

\subsection{Model Components}

In what follows, we describes in details the different components of the developed cc-commerce model, namely, client, provider, auditor, broker, security and privacy, and communication networks components. 


\subsubsection{CC-Commerce Client}

The cc-commerce client is the principal stakeholder for the cc-commerce provider. A client represents a user/organization that maintains a business relationship with, and uses the service from a provider. A client browses the list of services provided by a provider, requests the appropriate service, sets up service contracts with the provider, and uses the service. The client may be billed for the service provisioned, and needs to arrange payments accordingly.

Clients need SLAs to specify the technical performance requirements fulfilled by a provider. SLAs can cover terms regarding the QoS, security, remedies for performance failures. A provider may also list in the SLAs a set of promises explicitly not made to clients, i.e. limitations, and obligations that clients must accept. A client can freely choose a provider with better pricing and more favorable terms. Typically, a provider pricing policy and SLAs are non-negotiable, unless the client expects heavy usage and might be able to negotiate for better contracts. Depending on the services requested, the activities and usage scenarios can be different among clients. Clients have access to the services provided by the cc-commerce components and by the CSPs.

\subsubsection{CC-Commerce Provider}

A cc-commerce provider is a user/organization that is responsible for making a service available to interested parties. A provider acquires and manages the computing infrastructure required for providing the services, runs the cc-commerce software that provides the services, and makes arrangement to deliver the cloud services to the clients through the network. It can be seen in Figure 2 that the provider is basically a CSP. It has its own CSP, cloud auditor, cloud broker, and cloud carrier. In this case, the provider can access the same services provided by the cloud computing infrastructure, and provide these services to its client. A CSPs' activities can be described in four major areas, these are: service deployment, service administration, cloud service management, and security and privacy.

\subsubsection{CC-Commerce Auditor}

A cc-commerce auditor is a party that can perform an independent investigation of cc-commerce service controls with the aim to express an opinion thereon. Audits are performed to verify conformance to standards through review of objective evidence. An auditor can evaluate the services provided by a provider in terms of security controls, privacy impact, and other performance measures. Auditing is especially important for central agencies as agencies should include a contractual clause enabling third parties to assess security controls of cloud providers $[6,17]$.

Security controls are the management, operational, and technical safeguards or countermeasures employed within an organizational information system to protect the confidentiality, integrity, and availability of the system and its information. For security auditing, an auditor can make an assessment of the security controls in the information system to determine the extent to which the controls are implemented correctly, operating as intended, and producing the desired outcome with respect to the security requirements for the system. The security auditing should also include the verification of the compliance with regulation and security policy. For example, an auditor can be tasked with ensuring that the correct policies are applied to data retention according to relevant rules for the authority. The auditor may ensure that fixed content has not been modified and that the legal and business data archival requirements have been satisfied. A privacy impact audit can help law agencies comply with applicable privacy laws and regulations governing an individual's privacy, and to ensure confidentiality, integrity, and availability of an individual's personal information at every stage of development and operation. 


\subsubsection{CC-Commerce Broker}

As e-commerce evolves, the integration of cc-commerce services can be too complex for clients to manage. A client may request cc-commerce services from a cc-commerce broker, instead of contacting a cc-commerce provider directly. A broker is an entity that manages the use, performance and delivery of cc-commerce services and negotiates relationships between providers and clients. In general, a broker can provide services in three categories [6]:

- Service intermediation: A broker improves a specified service by enhancing some specific capability and providing value-added services to clients. The enhancement can be managing access to cloud services, identity management, performance reporting, enhanced security, etc.

- Service aggregation: A broker combines and integrates multiple services into one or more new services. It provides data integration and ensures secure data exchange between the clients and multiple providers.

- Service arbitrage: Service collection is similar to service aggregation except that the services being aggregated are not fixed. Service collection allows a broker to flexibly choose services from multiple agencies. The broker, for example, can use a credit-scoring service to measure and select an agency with the best score.

\subsubsection{CC-Commerce Security and Privacy}

In order to enable cloud-driven cc-commerce growth and innovation, we must provide strong information and network security measures. However, to do so, we must have a clear framing on what is meant by information and network security [25, 26]. The canonical goals of information security are: authentication, access control, data confidentiality, data integrity, and nonrepudiation. Furthermore, availability, accountability, assurance, and resilience, must be consider carefully considered [27]. It is hard to imagine a cc-commerce system running without using strong security and privacy, and one of the most powerful security protocols that has been developed is the secure socket layer (SSL) protocol $[28,29]$.

\subsubsection{Communications Network}

A communications network acts as an intermediary that provides connectivity and transport of cccommerce services between clients and providers. Communications networks provide access to clients through network, telecommunication and other access devices. For example, clients can obtain cc-commerce services through network access devices, such as computers, laptops, mobile phones, mobile Internet devices (MIDs), etc. The distribution of cc-commerce services is normally provided by network and telecommunication carriers or a transport agent, where a transport agent refers to a business organization that provides physical transport of storage media such as high-capacity hard drives. Note that a provider will set up SLAs with a communications network to provide services consistent with the level of SLAs offered to clients, and may require the communications network to provide dedicated and secure connections between clients and cloud providers.

\subsection{CC-Commerce Deployment Models}

The cc-commerce model developed in this work can be deployed using one of the following configurations: (1) Provider-access configuration, and (2) Broker-access configuration. In the provider-access configuration, a client directly accesses a cc-commerce provider, where the cccommerce infrastructure and computing resources are made available to the general public (clients) over a public network. The configuration can be owned by an organization selling cloud 
services or by an organization hiring cloud services and deploy its cc-commerce technology on top of it to serve a diverse pool of clients.

In the broker-access configuration, a client accesses a cc-commerce broker to accomplish a certain mission or query, the broker, in turn, analyze the query and choose the best path to accomplish the mission, which could be through intra-connection with local recourses (cccommerce 1) or through interconnection with other cc-commerce sites (cc-commerce 2, cccommerce $3, \ldots$, cc-commerce $n$ ). In this case, the broker either outsources the query to one or more cc-commerce sites or collaborates with other cc-commerce sites. The interconnection with other cc-commerce sites can be done through dedicated links or through existing Internet infrastructure. A special case of the broker-access configuration is that when collaborating sites host resources only for one enterprise or organization, then it can be considered as a c-commerce configuration.

For more efficient implementation, we highly recommend to host the cc-commerce auditor at the same site of the cc-commerce provider/broker. Furthermore, it is always recommended to host a cc-commerce broker and at least one provider at the same site. The security and privacy components must be installed at all sites. However, it is necessary to realize that installation of security and privacy components and interconnection through WAN links increase query processing time or response time. In other words, it is expected that provider-access configuration always performs faster than the broker access configuration, specially, if the broker outsources part or the entire requested query.

\section{ImPlementation and Performance Measures}

In order to evaluate the performance of the cc-commerce model described in previous sections, and compare its performance against conventional c-commerce models, we developed a test tool, which is referred to as 3CT tool for CC-Commerce Test tool. It can be used to evaluate the performance of the model through performing c-commerce and cc-commerce processes across the Internet. In particular, we subscribe for three Websites at three different hosting providers as described in Table 2.

Table 2. Public IP addresses of the sites.

\begin{tabular}{|c|l|l|l|}
\hline Site & \multicolumn{1}{|c|}{ Web Host Name } & \multicolumn{1}{c|}{ Web Host URL } & \multicolumn{1}{c|}{ Public IP address } \\
\hline DC1 & Ixwebhosting & http://www.ixwebhosting.com/ & 98.130 .174 .2 \\
\hline DC2 & WebsitePanel & http://ccp.my-hosting-panel.com/ & 204.93 .174 .60 \\
\hline DC3 & networksolutions & http://www.networksolutions.com/ & 205.178.152.126 \\
\hline
\end{tabular}

As a test data, the NorthWind.MDF database (http://northwinddatabase.codeplex.com) is uploaded to all those Websites to be used as a test data, where queries can be performed requesting a certain data from the NorthWind.MDF database as it will be explained in details in Section 6. NorthWind.MDF database can be downloaded as an executable SQL script (285 KB only) or as a backup that can be restored with sample data currently works with SQL Server 2005 and SQL Server 2008.

The 3CT tool can be used in provider or broker access configurations. It consists of a single Webpage interface through which we can initiate a request (query) for retrieving data from the uploaded NorthWind.MDF database. The 3CT tool is programmed using ASP.NET (C\#), LINQ to SQL Class, Microsoft Visual Studio 2008, C\# Web application, and MS SQL Express 2008 Server. The Webpage is hosted at the ixwebhosting Web hosting (URL: www.ixwebhosting.com, IP address: 98.130.174.2). It can be accessed at http://www.awad.texcept.com/default.aspx. 
In addition to the many advantages of cloud computing that can benefit from in cc-commerce, we believe that cc-commerce can have one other advantage which is the fast response as compared to c-commerce. In order to numerically evaluate the performance, we calculate the response time $(T)$, which is defined as the CPU time required retrieving the requested data from the various servers. Due to network instability, the response time for equivalent data retrieval tasks are usually measured more than once at different times during the day, and the average response time $\left(T_{\text {avg }}\right)$ and the associated standard deviation $(\sigma)$ should be calculated. The calculated $T_{\text {avg }}$ depends on:

- The amount of the retrieved data.

- The number of collaborating hosts including the main Website server.

- The network configuration.

- The bandwidth and delay of the data communication links between the client and the main server and between the main server and other collaborating servers.

To evaluate the performance of the provider-access configuration, the tool retrieves the data from the same hosting server; i.e., it represents a cc-commerce model. For broker-access configuration evaluation, the tool retrieves data from various hosting servers. However, since each hosting server is assumed to host data for a single enterprise or organization, then it can be considered as a c-commerce evaluation.

In c-commerce usually a client accesses a specific Website, which is called the main Website, for the sake of performing a certain task, for example, retrieving data stored at one or more servers (hosts) located at various locations that are internetworked using different WAN technologies and protocols and having various public IP addresses on the Internet. The server that hosts the main Website is called the main Server. In the new cc-commerce model, the main Web server and all collaborating servers are located at the same location under the same public IP address interconnected using LAN technologies and protocols. Thus, it is expected to reduce the response time significantly because of replacing the WAN data communication links between the main Web server and other collaborating servers by the high bandwidth and small delay LAN data communication links.

\section{RESUltS AND DisCUSSIONS}

This section uses 3CT tool described in Section 5 to evaluate and compare the performance of the new cc-commerce model against the performance of c-commerce models having different number of collaborating parties. In particular, four scenarios are considered, three of them represent different c-commerce configurations (broker-access configuration with one enterprise per site) and one of them represents a cc-commerce configuration (provider-access configuration). In all scenarios the response time for equivalent data retrieval tasks from the NorthWind.MDF database are measured more than once and the average response time $\left(T_{\text {avg }}\right)$ and the associated standard deviation $(\sigma)$ are calculated.

In all scenarios, a client accesses the MainWebServer at Site 1 requesting some data. Then, Site 1 server retrieves these data from its local data storage or from one or more other servers at other sites. In particular, in this work, to demonstrate and investigate the effect of the size of the retrieved data on the performance of the c-commerce system, we perform two queries requesting two different data sizes. The sizes of the requested data are $850 \mathrm{~KB}$ and $1958 \mathrm{~KB}$. For the 850 $\mathrm{KB}$, three entities from the NorthWind.MDF database are retrieved, namely, Customers (48 KB), Employees $(268 \mathrm{~KB})$, and Orders $(534 \mathrm{~KB})$. While for the $1958 \mathrm{~KB}$, five entities from the NorthWind.MDF database are retrieved, namely, Customers, Employees, Orders (twice from two different sites), and Orders Details (574 KB). 
The first scenario (Scenario \#1) simulates a c-commerce system that consists of three sites, which are hosted under three different public IP addresses, as shown in Figure 7. As it can be seen in this figure, Site 1 hosts the MainWebServer, which acts as a cc-commerce broker, and the other two sites act as collaborating sites. In other words, the MainWebInterface is acting as a broker outsourcing the execution of the received query to other sites. Each of the above sites (including the main site) includes a Web Server and SQL Server connected to data storages, where the NorthWind.MDF database is uploaded and stored. The IP addresses of these sites are given in Table 1. The details of the retrieved data (names of entities, sizes of entities, and number of records (rows) per entity), and the IP addresses of the sites from which they are retrieved are given in Table 2.

Table 2. Details of retrieved data from the NorthWind.MDF database (Scenario \#1).

\begin{tabular}{|c|c|c|c|c|c|c|c|c|c|c|c|c|}
\hline \multirow{3}{*}{ Entity } & \multicolumn{6}{|c|}{$850 \mathrm{~KB}$} & \multicolumn{6}{|c|}{$1958 \mathrm{~KB}$} \\
\hline & \multirow{2}{*}{$\begin{array}{c}\text { Size } \\
\text { (KB) }\end{array}$} & \multirow{2}{*}{ Rows } & \multicolumn{4}{|c|}{ Scenario } & \multirow{2}{*}{$\begin{array}{l}\text { Size } \\
(\mathbf{K B})\end{array}$} & \multirow{2}{*}{ Rows } & \multicolumn{4}{|c|}{ Scenario } \\
\hline & & & $\# 1$ & $\# 2$ & $\# \mathbf{3}$ & $\# 4$ & & & $\# 1$ & $\# 2$ & $\# \mathbf{3}$ & $\# 4$ \\
\hline Customers & 48 & 91 & DC3 & DC1 & DC2 & DC1 & 48 & 91 & DC3 & DC1 & DC2 & DC1 \\
\hline Employees & 268 & 9 & DC2 & DC2 & DC2 & DC1 & 268 & 9 & DC2 & $\mathrm{DC} 2$ & $\mathrm{DC} 2$ & DC1 \\
\hline Orders & 534 & 830 & DC3 & DC3 & DC2 & DC1 & 534 & 830 & $\mathrm{DC} 2$ & $\mathrm{DC} 2$ & $\mathrm{DC} 2$ & DC1 \\
\hline Orders & - & - & - & - & - & - & 534 & 830 & DC3 & DC3 & $\mathrm{DC} 2$ & DC1 \\
\hline $\begin{array}{l}\text { Order } \\
\text { Details }\end{array}$ & - & - & - & - & - & - & 574 & 2153 & DC3 & DC3 & $\mathrm{DC} 2$ & DC1 \\
\hline
\end{tabular}




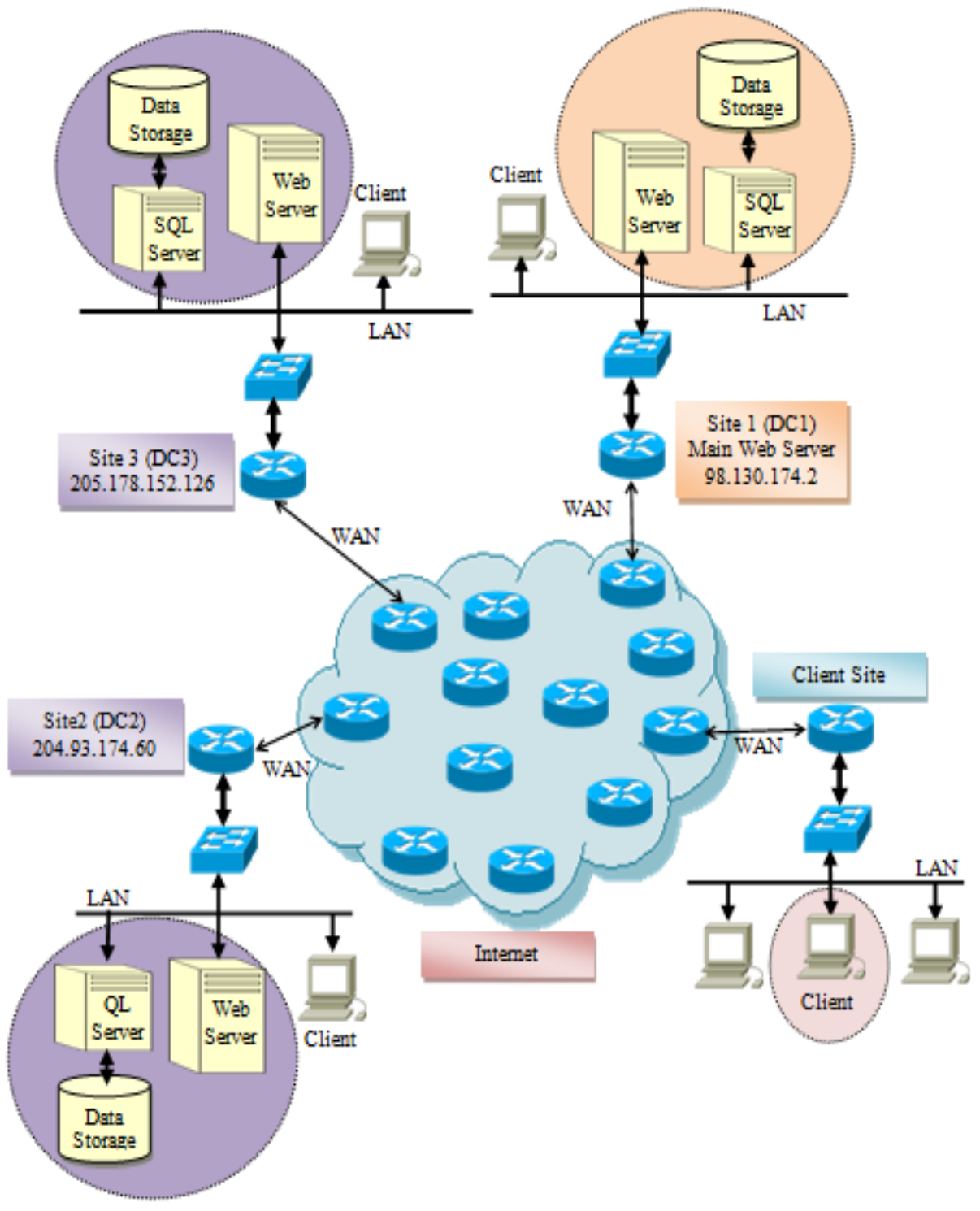

Figure 7. The network configuration.

The second scenario (Scenario \#2) simulates a c-commerce system similar to that in Scenario \#1 except, some of the data is retrieved from the data storage hosted at Site 1 (MainWebServer) at the public IP address 98.130.174.2. In particular, the data for the entity Customers (48 KB) is retrieved from the SQL Server at Site 1 (DC1), while the rest of the data is retrieved from the other sites as given in Table 2. The third scenario (Scenario \#3) simulates a c-commerce system similar to that in Scenario \#1 and \#2, in which the MainWebServer retrieves all data from one collaborating site at DC2 (public IP address 204.93.174.60). The sizes of data to be retrieved are $850 \mathrm{~KB}$ and $1958 \mathrm{~KB}$ similar to those in previous scenarios, which are also detailed in Table 2.

The fourth scenario (Scenario \#4) simulates a cc-commerce, in which a client accesses the MainWebInterface retrieved all data from its local storage. In this case, the MainWebInterface is acting as a cc-commerce provider. In particular, in this Scenario, the client requesting data from the MainWebServer at DC1 (public IP address 98.130.174.2), which realizes that all data is 
available locally so it does not need to collaborate with other sites in response for this query. The retrieved data is detailed in Table 2.

As, it has been discussed earlier that due to network instability, the response time for each data retrieving process is not constant and keeps changing. Therefore, in this work, we perform the data retrieving process 19 times, each time, the response time $\left(T_{i}\right)$, which is defined as the CPU time required retrieving the data from their destination addresses is calculated. Then, $T_{\text {avg }}$ and $\sigma$ are calculated. The results for $T_{i}, T_{\text {avg }}$, and $\sigma$ are given in Table 3. The table also shows another parameter which is the speedup factor $(S)$, which represents how much a cc-commerce runs faster than a certain c-commerce configuration. It is calculated by dividing the response time of the ccommerce configuration by the response time of the cc-commerce configuration. The results for $T_{\text {avg }}$ for all scenarios are shown in Figure 8.

Table 3. The run and average response times for Scenario \#1.

\begin{tabular}{|c|c|c|c|c|c|c|c|c|}
\hline \multirow{3}{*}{ Run } & \multicolumn{9}{|c|}{$\boldsymbol{T}_{\boldsymbol{i}}$ (msec) } \\
\cline { 2 - 9 } & \multicolumn{2}{|c|}{ Scenario \#1 } & \multicolumn{2}{|c|}{ Scenario \#2 } & \multicolumn{2}{c|}{ Scenario \#3 } & \multicolumn{2}{c|}{ Scenario \#4 } \\
\cline { 2 - 9 } & $\mathbf{8 5 0 ~ K B}$ & $\mathbf{1 9 5 8 ~ K B}$ & $\mathbf{8 5 0 ~ K B}$ & $\mathbf{1 9 5 8}$ KB & $\mathbf{8 5 0 ~ K B}$ & $\mathbf{1 9 5 8 ~ K B}$ & $\mathbf{8 5 0 ~ K B}$ & $\mathbf{1 9 5 8 ~ K B ~}$ \\
\hline 1 & 937.5 & 968.8 & 593.8 & 1140.6 & 640.6 & 890.6 & 109.4 & 156.3 \\
\hline 2 & 421.9 & 937.5 & 375.0 & 703.1 & 265.6 & 406.3 & 109.4 & 187.5 \\
\hline 3 & 406.3 & 1359.4 & 390.6 & 1031.3 & 234.4 & 421.9 & 78.1 & 109.4 \\
\hline 4 & 453.1 & 875.0 & 421.9 & 656.3 & 250.0 & 406.3 & 171.9 & 234.4 \\
\hline 5 & 531.3 & 1171.9 & 562.5 & 1000.0 & 234.4 & 390.6 & 140.6 & 218.8 \\
\hline 6 & 421.9 & 1078.1 & 687.5 & 671.9 & 234.4 & 390.6 & 78.1 & 109.4 \\
\hline 7 & 671.9 & 843.8 & 437.5 & 953.1 & 359.4 & 531.3 & 125.0 & 171.9 \\
\hline 8 & 718.8 & 781.3 & 390.6 & 1093.8 & 234.4 & 390.6 & 109.4 & 156.3 \\
\hline 9 & 515.6 & 734.4 & 359.4 & 734.4 & 250.0 & 406.3 & 109.4 & 140.6 \\
\hline 10 & 500.0 & 1000.0 & 406.3 & 796.9 & 234.4 & 375.0 & 140.6 & 218.8 \\
\hline 11 & 578.1 & 953.1 & 359.4 & 781.3 & 234.4 & 390.6 & 78.1 & 125.0 \\
\hline 12 & 765.6 & 937.5 & 546.9 & 1031.3 & 234.4 & 390.6 & 93.8 & 125.0 \\
\hline 13 & 1187.5 & 734.4 & 343.8 & 1765.7 & 953.1 & 1171.9 & 671.9 & 843.8 \\
\hline 14 & 484.4 & 859.4 & 390.6 & 750.0 & 281.3 & 437.5 & 125.0 & 187.5 \\
\hline 15 & 406.3 & 750.0 & 343.8 & 593.8 & 250.0 & 390.6 & 140.6 & 203.1 \\
\hline 16 & 390.6 & 921.9 & 437.5 & 593.8 & 250.0 & 406.3 & 109.4 & 156.3 \\
\hline 17 & 687.5 & 953.1 & 359.4 & 1359.4 & 250.0 & 406.3 & 187.5 & 218.8 \\
\hline 18 & 562.5 & 859.4 & 421.9 & 765.6 & 250.0 & 406.3 & 78.1 & 125.0 \\
\hline 19 & 750.0 & 1406.3 & 1000.0 & 1078.1 & 1015.6 & 1281.3 & 343.8 & 468.8 \\
\hline$T_{\text {avg }}$ & 599.52 & 953.96 & 464.65 & 921.07 & 350.34 & 520.57 & 157.90 & 218.77 \\
$(\sigma)$ & $(207.75)$ & $(188.55)$ & $(160.68)$ & $(294.10)$ & $(242.29)$ & $(274.62)$ & $(138.29)$ & $(170.94)$ \\
\hline$S$ & 3.80 & 4.36 & 2.94 & 4.21 & 2.22 & 2.38 & 1.00 & 1.00 \\
\hline
\end{tabular}

Figure 8. Comparison of average 


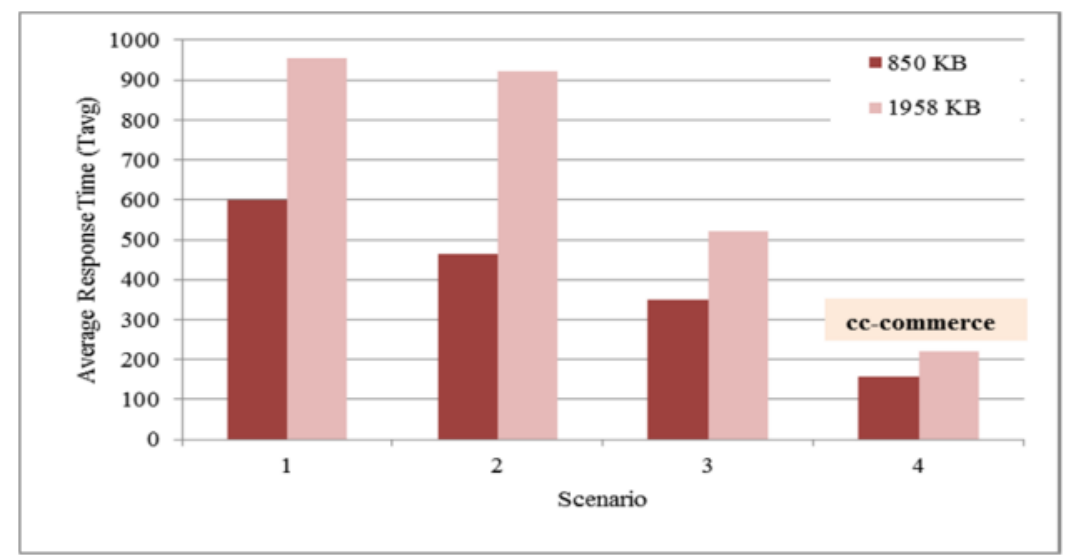

sponses times $\left(T_{\text {avg }}\right)$ for all scenarios.

\section{CONCLUSIONS}

This paper presents a description and performance analysis of a new cc-commerce model, which can be deployed using two configurations, namely, provider-access configuration and brokeraccess configuration. The new cc-commerce model consists of six main components, namely: client, provider, auditor, broker, security and privacy, and communications network. The new model is implemented and a simple tool called cc-commerce test (3CT) tool is developed to evaluate the performance of the new cc-commerce model through a number of scenarios. In these scenarios, various systems configurations are considered representing c-commerce and cccommerce configurations. For each configuration, the average response times for retrieving different sets of data from the NorthWind.MDF database are calculated. Then, the speedup factor achieved by the cc-commerce configuration over the various c-commerce configurations are computed and discussed.

The main conclusions of this work can be summarized as follows:

(1) Cloud computing can play a big role in providing cost-effective computing resources for businesses willing to use e-commerce or be part of c-commerce systems. Especially, small and medium businesses who cannot afford the cost of installing on-premises computing resources.

(2) Cloud computing can be used as an alternative to the on-premises computing resources forming a high-performance cloud computing based c-commerce or cc-commerce. It eliminates the drawbacks of c-commerce (e.g., installation and running costs, delay, security, etc.).

(3) The developed cc-commerce model with both provider-accessed and broker-accessed configurations can be used in porting any c-commerce application to a powerful and a costeffective cc-commerce application.

(4) The average response time of a cc-commerce application is always less than the average response time of its equivalent c-commerce application. Furthermore, the cc-commerce application provides better high availability, scalability, reliability, and consequently, more clients and applications satisfactions.

(5) The four scenarios examined in this paper demonstrate that for the equivalent tasks a speedup factor of more than 2 can be achieved using a cc-commerce configuration over a ccommerce configuration.

(6) The results obtained should be very encouraging for all enterprises (specially small and medium enterprises) to go for cloud computing as a common computing resource for ecommerce (i.e., using cc-commerce business model). 
International Journal on Cloud Computing: Services and Architecture (IJCCSA),Vol.3, No.1, February 2013

The main recommendations for future work and research may include using $3 \mathrm{CT}$ tool to perform further investigations, in particular, retrieve more data from more sites, and also evaluate the performance of broker-access cc-commerce configuration with more than one enterprise per site against the performance of provider-access cc-commerce configuration, considering equal retrieved data and collaborating enterprises.

\section{REFERENCES}

[1] G. Schneider (2010). Electronic commerce. Learning Publisher.

[2] M. Khosrowpour (2004). The Social and Cognitive Impacts of E-Commerce on Modern Organizations. IGI-Global Publisher.

[3] M. Khosrowpour (2006). Cases on Electronic Commerce Technologies and Applications. IGI-Global Publisher.

[4] B. A. Forouzan (2007). Data Communications and Networking (4th Edition). McGraw-Hill.

[5] M. Chen, D. Zhang, \& L. Zhou (2007). Empowering Collaborative Commerce with Web Services Enabled Business Process Management Systems. Decision Support Systems, Vol. 43, pp. 530-546.

[6] T. Velte, A. Velte, R. Elsenpeter (2009). Cloud Computing: A practical Approach. McGraw-Hill.

[7] L. Wu \& Y. Duan (2006). Research on Collaborative Commerce Model Based on Web Services. Proceedings of the 4th International Conference on Electronic Business (ICEB2004), Beijing, China, 5-9 December 2004, pp. 661-664.

[8] C. Peiris, D. Sharma, \& B. Balachandran (2011). C2TP: A Service Model for Cloud. International Journal of Cloud Computing, Vo. 1, No. 1, pp. 3-22.

[9] F. H. Chanchary \& S. Islam (2012). Cloud Commerce: Readiness Measurement of KSA. International Journal of Wireless Information Networks \& Business Information System, Vol. 2, Issue March/April, pp. 70-81.

[10] T. Issa, V. Chang, \& T. Issa (2010). The Impact of Cloud Computing and Organizational Sustainability. Proceedings of the 1st International Conference on Cloud Computing and Virtualization (CCV'10), Singapore, 3-4 May 2010, pp.163-169.

[11] Q. Li \& N. Xia (2011). The Utilization of Cloud Computing in Network Collaborative Commerce Chain. Proceedings of the 4th International Conference on Business Intelligence and Financial Engineering (ICBIFE'11), Wuhan, Hubei China, 17-18 October 2011, pp. 279-284.

[12] B. Thuraisingham, A. Gupta, E. Bertino, \& E. Ferrari (2002). Collaborative Commerce and Knowledge Management. Knowledge and Process Management, Vol. 9, No. 1, pp. 43-53.

[13] Nerve Wire (2002). Collaborative Commerce: Compelling Benefits, Significant Obstacles. Retrieved from http://www.nervewire.com/about/press_archive/ collab_comm_survey.htm on 14th September, 2012.

[14] E. Turban, J. K. Lee, D. King, T. P. Liang, \& D. Turban (2010). Electronic Commerce. Prentice-Hall.

[15] R. Basmadjian, H. D. Meer, R. Lent, \& G. Giuliani (2012). Cloud Computing and Its Interest in Saving Energy: The Use Case of a Private Cloud. Journal of Cloud Computing: Advances, Systems and Applications Vol. 1, No. 5.

[16] CrownPeak. Retrieved from http://www.crownpeak.com/downloads/white-papers/on-premise-vscloud-the-impact-of-cloud-computing-on-web-content-management.pdf on 17th February 2013.

[17] A. Goscinski \& M. Brock (2011). Toward Higher Level Abstractions for Cloud Computing. International Journal of Cloud Computing, Vol. 1, No. 1, pp. 37-57.

[18] R. Buyya, C. S. Yeo, S. Venugopala, J. Broberg, \& I. Brandic (2009). Cloud Computing and Emerging IT Platforms: Vision, Hype, and Reality for Delivering Computing as the 5th Utility. Future Generation Computer Systems, Vol. 25, No. 6, pp. 599-616.

[19] P. Sasikala (2011). Cloud Computing: Present Status and Future Implications. International Journal of Cloud Computing, Vol. 1, No. 1, pp. 23-36.

[20] T. Hartman (2009). Getting Started with Collaboration Services and the Cloud. Retrieved from http://www.avanade.com/Documents/Research\%20and\%20Insights/gettingstartedwithcollaborationse rvicesandthecloud477077.pdf on 17th February 2013.

[21] S. Huang \& Y. Fan (2007). Strategy, Model, and Platform for Collaborative Commerce. Proceedings of the IEEE International Conference on E-Business Engineering (ICEBE'07), Hong-Kong, China, 24-26 October 2007, pp. 304-311. 
[22] M. Zhang, Y. Chen, J. Y. Lai (2008). Design of the Collaborative Commerce System Model: An Application Issue in Theme Park of China. Proceedings of the IEEE International Conference on Industrial Engineering and Engineering Management (IEEM '08), China, 8-11 December 2008, pp. 1465-1468.

[23] H. H. Chang \& I. C. Wang (2011). Enterprise Information Portals in Support of Business Process, Design Teams and Collaborative Commerce Performance. International Journal of Information Management, Vol. 31, No. 2, pp. 171-182.

[24] R. R. Exposito, G. L. Taboada, S. Ramos, J. Tourino, \& R. Doallo (2013). Performance Analysis of HPC Applications in the Cloud. Future Generation Computer Systems, Vol. 29, No. 1, pp. 218-229.

[25] W. a. Jansen (2011). Cloud Hooks: Security and Privacy Issues in Cloud Computing. Proceedings of the 44th Hawaii International Conference on System Sciences (HICSS'11), Hawaii, USA, 4-7 January 2011.

[26] T. Mather, S. Kumaraswamy, \& S. Latif (2009). Cloud Security and Privacy: An Enterprise Perspective on Risks and Compliance (Theory in Practice). O'Reilly Media.

[27] D. Chen (2012). Data Security and Privacy Protection Issues in Cloud Computing. Proceedings of the 2012 International conference on Computer Science and Electronics Engineering (ICCSEE'12), Hangzhou, China, 23-25 March 2012, Vol. 1, pp. 647-651.

[28] A. Freier, P. Karlton, \& P. Kocher (2011). The Secure Sockets Layer (SSL) Protocol Version 3.0. IETF RFC 6101. Retrieved from http://tools.ietf.org/html/rfc6101 on October, 2012.

[29] Z. Hu (2011). The Study of E-commerce Security Protocol. Proceedings of the 2011 International Conference on Intelligence Science and Information Engineering (ISIE '11), Wuhan, China, 20-21 August 2011, pp. 349-352.

\section{AUTHORS}

Hussein Al-Bahadili (hbahadili@uop.edu.jo) is an associate professor at Petra University. He received his $\mathrm{PhD}$ and M.Sc degrees from University of London (Queen Mary College) in 1991 and 1988. He received his B.Sc in Engineering from the University of Baghdad in 1986. He is a visiting researcher at the Centre of Wireless Networks and Communications (WNCC) at the School of Engineering, University of Brunel (UK). He has published many papers in different fields of science and engineering in numerous leading scholarly and practitioner journals, and presented at leading world-level scholarly conferences. He has published three chapters in

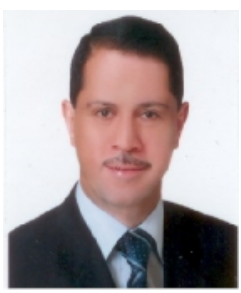
prestigious books in IT and Simulations. He is also a reviewer for a number of books, and currently, he is an editor of a book on Simulation in Computer Network Design and Modeling: Use and Analysis. His research interests include computer networks design and architecture, routing protocols optimizations, parallel and distributed computing, cryptography and network security, data compression, software and Web engineering.

Mr. Awad Al Sabbah (awadsabbah@ hotmail.com) is a computer trainer and instructor in Kuwait Ministry of Education, Kuwait. He received his B.Sc degree in computer science from Yarmouk University, Jordan in 2004, and his M.Sc degree in computer information systems from Middle-East University, Amman, Jordan in 2012 under the supervision of Dr. Hussein Al-Bahadili. His current research interests are in developing and analysis cloud collaborative commerce models, cloud systems, e- commerce, Webbased applications.

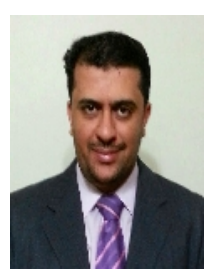

Dr. Mohammed Abu-Arqoub (abu-arqoub@uop.edu.jo) is an assistant professor at the Faculty of Information Technology, Petra University, Amman, Jordan, and he is acting as the Head of the Computer Science Department. He earned his M.Sc and PhD from the University of Saint-Petersburg Electro-Technical University (Russia) in 1990 and 1995, respectively. He has published many papers at world level scholarly conferences and international journals. His research interests include data structure, computer, Web-based system design, e-commerce, computer simulation, computer architecture, database optimization, parallel and distributed computing, middleware systems, software and Web engineering.

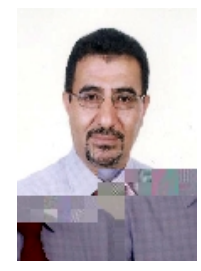

